\title{
Machine Learning
}

\section{Based Cost Engineering}

\section{of Automotive Parts -}

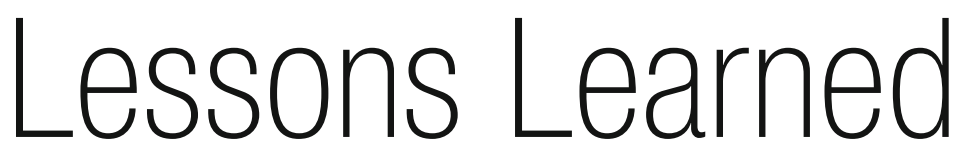

\section{Frank Bodendorf, Jörg Franke}

Strategic change management is one of the crucial fields of cost analytics in the automotive industry. Here, one important task is to explore potentials and propose measures to reduce costs of parts in the product development process. This is usually done within cost engineering and controlling departments of car manufacturing companies. The costs of part modifications are analyzed and specified usually by complex calculation methods based on a lot of input parameters describing the properties of a specific part. The calculation processes are mostly executed bottom-up. This means that the cost of a part is determined by going through many attributes and assigning and accumulating the corresponding cost estimations. These attributes comprise features of CAD designs, built-in materials as well as production sequences.

That is why the traditional calculation processes are effortful and time consuming. In addition, they are predominantly carried out manually with the help of simple calculation software like spreadsheets. Actually, Machine Learning (ML) algorithms and models are occupying center stage to cope with this problem and make the process more efficient. The overall goal is to predict costs of parts in the early phase of the product development process. In this early phase the expenditure of part modifications is low, but detailed information on the structure of the part is scarce. Machine Learning models are expected to be able to learn from a large amount of historical data and predict costs without using bottom-up calculation formulas. Also, Machine Learning algorithms are expected to find out the most influencing technical and economic parameters and in this way are able to reduce the considered input data to factors that are at hand in the early stage of the development process.

The figure below outlines the procedure model of a series of studies that has been conducted at a large automotive

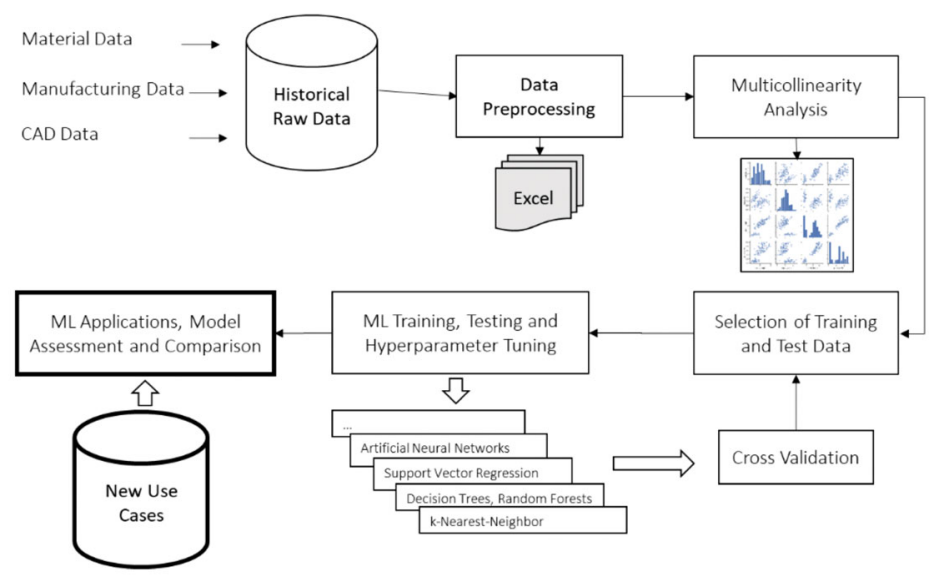

manufacturer in the state of Bavaria, Germany. These studies aim at developing different ML models to predict modification costs of automotive parts in an efficient way by learning from historical calculation data and results. The investigated ML models comprise Multilinear Regression, Artificial Neural Network, Support Vector Regression, Decision Tree, Random Forest, and k-Nearest Neighbor.

The developed ML models have been applied to a sample of use cases with given input parameters but unknown cost. In parallel, the use cases have been calculated traditionally and manually to provide traditional cost estimations. The ML models are then assessed and compared in regard to their predictive power and accuracy. At the same time, it is looked at the reduced input parameter set used and investigated how those input parameters can be obtained in the early phase of product development. The ML applications will be demonstrated and the results of the comparative studies will be explained. 